Journal of

\section{Applied Crystallography}

ISSN 0021-8898

Received 2 November 2003

Accepted 25 March 2004

\title{
A modified microdialysis button for use in protein crystallization
}

\author{
Stephen S. J. Lee ${ }^{\mathrm{a} *}$ and Robert Cudney \\ ${ }^{a}$ The Division of Structural Biology, The Wellcome Trust Centre for Human Genetics, Roosevelt Drive, Oxford \\ OX3 7BN, UK, and ${ }^{\mathbf{b}}$ Hampton Research, 34 Journey, Aliso Viejo, CA 92656-3317, USA. Correspondence e-mail: \\ stephenl@strubi.ox.ac.uk or slee202020@hotmail.com
}

A modified microdialysis button for use in protein crystallization has been designed and manufactured. This new apparatus, called the Microdialysis Rod, allows easier handling and storage, and provides versatility to the users.

(C) 2004 International Union of Crystallograph Printed in Great Britain - all rights reserved

\section{Introduction}

Biological macromolecules crystallize in solution when an appropriate degree of supersaturation is reached. There are a number of devices, procedures and methods for altering the degree of saturation of a protein solution for this purpose. One method for obtaining protein crystals is dialysis, which can be set up in various ways, for example, in dialysis bags, Zeppezauer cells (Zeppezauer et al., 1968) or buttons. The Microdialysis Buttons, also known as Cambridge buttons, which were first employed for the crystallization of tyrosyltRNA synthetase (Reid et al., 1973), offer a convenient way to perform the dialysis crystallization method with a small amount of sample in a relatively convenient format. A protein sample is placed inside a small chamber on top of the button, which is then covered with a dialysis membrane. Since the dialysis membranes are semipermeable, they allow small-molecular-weight substances to diffuse in while preventing the protein from diffusing out (Ducruix \& Giegé, 1999; McPherson, 1999). Numerous proteins have been crystallized using these buttons; however, users often find them difficult to work with because of their relatively small size (they are small cylindrical devices, approximately $1 \mathrm{~cm}$ in diameter and $1 \mathrm{~cm}$ in height).

We present here a novel microdialysis device for protein crystallization, a modified version of the Microdialysis Buttons, which has been designed to fit into the well of a 24-well VDX Plate or a 24-well Linbro Plate. The Microdialysis Rods are compatible with the hanging-drop and dialysis techniques, will improve handling and storage, and will give versatility to users.

\section{Design specifications}

The Microdialysis Rods have a longer profile than the Microdialysis Buttons; the Microdialysis Rods are machined from transparent Perspex, but they can also be made by injection moulding (Fig. 1). The button end of the rod has a flat-bottom sample chamber, which is available in a 5, 10, 50 or $100 \mu \mathrm{l}$ volume. Like the Microdialysis Buttons, Microdialysis Rods use a rubber O-ring to secure a section of wet dialysis membrane (having the appropriate molecular-weight cut-off; MWC) over the mother-liquor-filled chamber; the O-ring in turn is held in place by seating it in the groove on the rod. Unlike the buttons, however, the Microdialysis Rods have been designed specifically to fit into the wells of certain 24 -well tissue culture plates. A circular flange has been strategically placed, between the button end and the handle, to prevent the rod from falling into the well completely. A second, smaller, flange/shoulder (proximal to the button end) makes sure that the rod is centred in the well. The Microdialysis Rods are currently available from Hampton Research.

\section{Applications}

A typical dialysis experiment involves taking a sample from the presence of a high ionic strength solution to a lower ionic strength solution (however, the technique can just as easily be used to proceed from low ionic strength to a higher ionic strength). This process is accomplished by placing the sample in high ionic strength in the Microdialysis Rod, sealing the rod with a dialysis membrane and then placing the sealed rod in a solution of ionic strength lower than that inside the rod. Salts, ligands and compounds smaller than the pore size of the dialysis membrane will leave the rod as long as their concentration is lower on the opposite side of the membrane. Once the concentration of the diffusible species is the same on both sides of the membrane, the system is in equilibrium.

When the protein sample is placed inside the Microdialysis Rod chamber, users should add enough to create a slight dome of liquid at the top edge of the button and use a 'Golf Tee Applicator' or a 'CRE Applicator' (available from Cambridge Repetition Engineers and Hampton Research) to seal the sample-filled chamber with a dialysis membrane. Following these guidelines will reduce the likelihood of trapping air bubbles between the sample solution and the membrane, which impedes dialysis. Furthermore, while adding the sample and applying the dialysis membrane, it helps to have the rod inserted, button-end up, in the well of a 24-well tissue culture plate for stability. Likewise, when it is time to recover the crystals, the rod should be mounted inside the well, the dialysis membrane should be removed (we found it best to leave the O-ring secured against the membrane; a surgical knife can be used to make an incision on the membrane around the outer circumference of the chamber opening and tweezers can then be used to remove the cutout by sliding it gently across the chamber opening; this method seems to minimize sample perturbation) and finally the crystal can be retrieved using a fibre loop of appropriate size; this whole procedure can be performed while viewing the rod under a light microscope.

During the fitting of the Microdialysis Rod into the well of a 24-well tissue culture plate for dialysis, the edge of the reservoir or well should have a bead of high-vacuum grease applied in a circular fashion to prevent evaporation. The individual plate reservoir should contain between 0.5 and $1.7 \mathrm{ml}$ of dialysis solution, completely covering the end of the Microdialysis Rod and wetting the dialysis membrane. It is important to keep the very end of the Microdialysis 


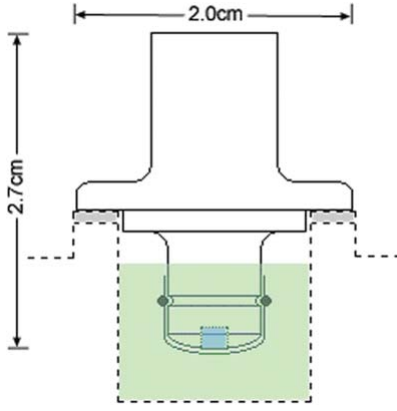

Figure 1

Schematic diagram of the Microdialysis Rod. Its overall dimensions are labeled. The two black circles represent the cross-sectional view of the O-ring, and the dialysis membrane is shown as a U-shaped line intersecting the two circles. The vacuum grease is coloured grey, the precipitant in the reservoir green and the protein sample in the rod chamber blue. The dotted line represents the crosssectional view of a single 24-well tissue culture plate well.

Rod with the membrane from becoming exposed to air or drying out during dialysis. Once the sample is placed in the Microdialysis Rod, the dialysis membrane applied, the reservoir edge greased and the reagent added to the reservoir, an even seal between the rod and the plate can be achieved by positioning the dialysis rod over the reservoir and then gently pressing the rod onto the reservoir with a twist. A plate cover or additional plates containing the Microdialysis Rods can be stacked on top of each other as long as at least four Microdialysis Rods are placed in the four corner wells of the plate. To monitor protein crystal growth, the Microdialysis Rod containing a sample can be viewed under a light microscope while it still rests in the plate reservoir. It should be pointed out that any plastic material (including the dialysis membrane) has some inherent birefringence that may make it difficult to determine if a crystal is birefringent or not (Harlos, 1992); however, on the basis of our crystallization experiments involving glucose isomerase (Fig. 2) and lysozyme (figure not shown), protein crystals can be observed clearly under a light microscope while the sample is in the rod assembly, and the residual birefringence from the plastic and membrane seems to be minimal (see Fig. 2).

In addition to dialysis, the Microdialysis Rods can be used in a hanging-drop vapour-diffusion experiment. A standard hanging-drop protocol is followed $(\sim 5,10,50$ or $100 \mu$ protein sample volume; depending on the rod used, against $\sim 200-350 \mu \mathrm{l}$ precipitant in the reservoir, making sure that it is not in contact with the sample drop), except that, in place of a cover slip, the protein sample is added to the chamber on the Microdialysis Rod. Instead of sealing the samplefilled chamber with a dialysis membrane, the chamber is left open. The sample-containing rod is inserted into the reservoir containing the appropriate precipitant, and the rod is sealed using vacuum grease. The protein crystal can be monitored and recovered (without the need to remove any dialysis membrane) as described above, under a similar viewing quality as is shown in Fig. 2, with less residual birefringence since a dialysis membrane is not employed.

\section{Advantages of the rod}

In addition to all the advantages that dialysis techniques can offer, such as keeping the protein concentration constant during the experiment (which the vapour-diffusion method cannot), allowing the

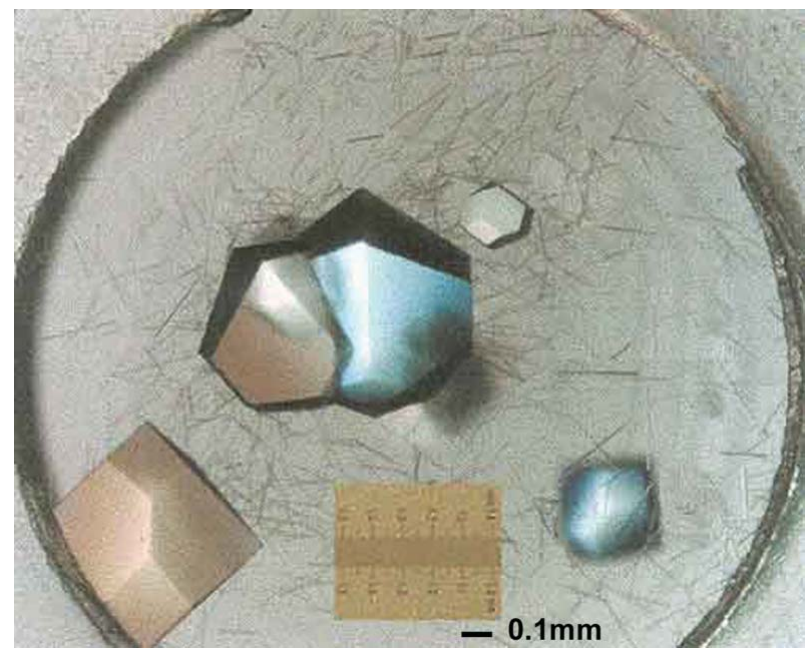

Figure 2

Crystals of glucose isomerase grown in the Microdialysis Rod. $2.5 \mu \mathrm{lof} 30 \mathrm{mg} \mathrm{ml}^{-1}$ glucose isomerase in $6 \mathrm{~m} M$ Tris, $\mathrm{pH} 8.4$, was added to the sample chamber and was dialyzed against $500 \mu \mathrm{l}$ of $1.5 \mathrm{M}$ ammonium sulfate at $\mathrm{pH} 8.4$, room temperature.

user to expose their sample to a continuum of potential crystalproducing conditions without physically perturbing the mother liquor and being able to control the rate of diffusion through the dialysis membrane by utilizing membranes with a particular molecular-weight cut-off (Ducruix \& Giegé, 1999; McPherson, 1999), the novel design of the Microdialysis Rod gives the user additional benefits. For instance, with their longer profile, the rods are easier to handle than the buttons. Since it has been specifically designed to fit many 24-well tissue culture plates, it is easier to transfer to other wells during or after the crystallization experiment, if necessary. Incorporating the plates also improves storage and record keeping. The plates housing the Microdialysis Rods can be easily stacked on top of each other in a space-saving manner, and since the wells come labeled on most plates it is easier to keep track of the crystallization conditions being tested. Furthermore, the Microdialysis Rod is a versatile protein crystallization tool; it can be reused indefinitely in both microdialysis and hanging-drop experiments. In some cases, where the protein is prone to the formation of large numbers of smaller crystals as a result of a rapid equilibration across a steep concentration gradient, a dialysis membrane having a smaller MWC or a double dialysis method (Thomas et al., 1989) can be utilized to reduce the rate of equilibration. In the double dialysis method, a Microdialysis Rod is prepared and placed inside a reservoir sealed with a dialysis membrane, which is in turn placed inside another reservoir.

\section{References}

Ducruix, A. \& Giegé, R. (1999). Editors. Crystallization of Nucleic Acids and Proteins. A Practical Approach. Oxford: IRL Press.

Harlos, K. (1992). J. Appl. Cryst. 25, 536-538.

McPherson, A. (1999). In Crystallization of Biological Macromolecules. New York: Cold Spring Harbor Laboratory Press.

Reid, B. R., Koch, G. L., Boulanger, Y., Hartley, B. S. \& Blow, D. M. (1973). J. Mol. Biol. 80, 199-201.

Thomas, D. H., Rob, A. \& Rice, D. W. (1989). Protein Eng. 2, 489-491.

Zeppezauer, M., Eklund, H. \& Zeppezauer, E. S. (1968). Arch. Biochem. Biophys. 126, 564-573. 